\title{
Recent Results and Status of EXO-200 and the nEXO Experiment
}

\author{
Caio Licciardi*, for the EXO-200 and nEXO Collaborations \\ Physics Department, Carleton University, Ottawa, ON, Canada \\ E-mail: licciardephysics.carleton.ca
}

\begin{abstract}
The EXO-200 experiment is searching for neutrinoless double beta decay using a time projection chamber filled with liquid xenon enriched in ${ }^{136} \mathrm{Xe}$. The detector has demonstrated exceptional performance with world leading measurements and stringent search results. The detector finished its Phase-I running shortly after an unexpected interruption in operations caused by incidents at the Waste Isolation Pilot Plant, but has restarted in Phase-II with hardware upgrades. During the two-year hiatus, the EXO-200 collaboration published several results using data from Phase-I. This article reports on the recent results and status of this detector, as well as on the proposed nEXO detector, the next generation experiment rooted in the success of EXO-200.
\end{abstract}

38th International Conference on High Energy Physics

3-10 August 2016

Chicago, USA

\footnotetext{
* Speaker.
} 


\section{Introduction}

Nuclear double beta $(\beta \beta)$ decay provides a promising possibility to probe physics beyond the Standard Model (SM), especially through the search of its hypothetical neutrinoless mode $(0 \mathrm{v} \beta \beta)$. In this mode, two nucleons simultaneously beta decay with emission of two electrons and nothing else. This process does not conserve lepton number and, if observed, would also imply that neutrinos are Majorana particles, i.e. their own antiparticles [1].

The $\beta \beta$ decay with emission of two antineutrinos and two electrons $(2 v \beta \beta)$ is an allowed process by the SM, that can only be observed if the single beta decay is strongly suppressed or forbidden by energy conservation. In the search for $0 v \beta \beta$, liquid ${ }^{136} \mathrm{Xe}(\mathrm{LXe})$ is a particularly appropriate choice of material satisfying that condition for various reasons, including:

- Xenon, being a noble element, is easy to purify and can be continuouly purified to achieve excellent chemical purity and cleanliness;

- ${ }^{136} \mathrm{Xe}$, being a gas at standard temperature and pressure thus easy to process in ultracentrifuges, is economical to enrich from the natural isotopic abundance of $8.9 \%$;

- the high Q-value of $2 v \beta \beta$ in ${ }^{136} \mathrm{Xe}, 2457.8 \mathrm{keV}$ [2], is among the largest and its half-life is the longest known, minimizing the contribution of backgrounds;

- LXe can be used both as the source and detection medium, maximizing efficiency and providing impressive self-shielding in monolithic detectors.

The EXO-200 collaboration has pioneered the use of large LXe detectors to search for $\beta \beta$ decays in ${ }^{136} \mathrm{Xe}$ with the discovery [3] and most precise measurement of the $2 v \beta \beta$ half-life [4], as well as setting stringent limits on $0 v \beta \beta$ [5]. Using $\sim 110 \mathrm{~kg}$ of active volume, it has demonstrated key performance parameters for the next generation of experiments that will search for $0 v \beta \beta$, such as the discrimination of $\gamma$-induced backgrounds based on event topology and high energy resolution. Recent results of this detector are discussed in section 2. Moving forward, nEXO is a proposed tonne scale experiment that builds on the technology demonstrated by EXO-200. It plans to incorporate new advancement in photosensors, charge collection tiles, and low temperature electronics. Exploiting ulta-low background materials, the sensitivity of nEXO is projected to probe the effective Majorana neutrino mass in the inverted hierarchy region. The nEXO experiment is briefly described in section 3 .

\section{The EXO-200 Experiment}

The EXO-200 detector is located in the Waste Isolation Pilot Plant (WIPP), Carlsbad, NM. It consists of two back-to-back cylindrical single-phase time projection chambers (TPCs), sharing a central cathode, filled with LXe that is enriched to $80.6 \%$ in the isotope ${ }^{136} \mathrm{Xe}$. The TPCs are contained in an ultra-low background copper vessel $\sim 44 \mathrm{~cm}$ long and $\sim 40 \mathrm{~cm}$ in diamater. Near the ends of the vessel is the readout instrumentation of each TPC, composed of a pair of wire planes, crossed at $60^{\circ}$, in front of an array of large-area avalanche photodiodes (APDs). Particles interacting with the LXe deposit energy that produces both scintillation light $(178 \mathrm{~nm})$, detected by the APDs almost instantenously, and electron-ion pairs. These electrons are drifted towards the 
wire grids inducing signals in the front-most wire plane ( $\mathrm{V}$-wires), and then collected by the second wire plane (U-wires). A more detailed description of the detector can be found elsewhere [6].

Information from the wires provide $2 \mathrm{D}$ reconstruction capability. The third dimension is obtained from the drift velocity in combination with the difference between light and charge detection times. This 3D event topology provides strong separation between single-site (SS) signal-like events, primarily $\beta$ or $\beta \beta$ decays, and multiple-site (MS) backgrounds, arising mostly from $\gamma$ interactions. Furthermore, the scintillation and ionization signals are combined to improve the energy resolution.

The EXO-200 Phase-I data acquisiton (DAQ) period spans between the start of its physics data taking in June, 2011, and an underground fire followed by radiation release at WIPP in February, 2014. During this period, the detector was successfully operated and the corresponding data used in numerous publications that are discussed in section 2.1. In early 2015, after restoration of regular access to the experimental area and detector operations, the EXO-200 Phase-II DAQ period started with hardware improvements presented in section 2.2.

\subsection{Phase-I Results}

Using the first two years of data, with a total ${ }^{136} \mathrm{Xe}$ exposure of $100 \mathrm{~kg} \cdot \mathrm{yr}$, the collaboration achieved an experimental sensitivity to the $0 v \beta \beta$ half-life of $1.9 \cdot 10^{25} \mathrm{yr}$, setting a limit with a profile likelihood scan of $\mathrm{T}_{1 / 2}^{0 v \beta \beta}>1.1 \cdot 10^{25} \mathrm{yr}$ at $90 \%$ confidence level (CL) [5]. In support of this analysis, the radioactivity-induced [7] and cosmogenic backgrounds [8] were studied in detail. The power of EXO-200, as a precision instrument, is corroborated by the measurement of $2 v \beta \beta$ decay in ${ }^{136} \mathrm{Xe}, \mathrm{T}_{1 / 2}^{2 v \beta \beta}=2.165 \pm 0.016$ (stat.) \pm 0.059 (sys.) $\times 10^{21} \mathrm{yr}[4]$. This has been the longest half-life observed and the most precisely measured $2 v \beta \beta$ decay half-life of any isotope to date.

The collaboration performed a search to alternative neutrinoless $\beta \beta$ decay modes, where one or two additional bosons are emittted along with the two electrons after the decay. These bosons are usually referred to as "Majorons". With improved understanding of the detector, the techniques employed in this analysis followed closely those used in previous papers, where the spectral shape of energy and spatial distribution constrain the backgrounds. The variable representing the spatial distribution of events was the standoff distance, defined as the distance between a charge deposit and the closest material that is not LXe, other than the cathode. No significant evidence of Majoron particles was found and limits on the coupling constants are among the strongest to date [9].

The $2 v \beta \beta$ decay of ${ }^{136} \mathrm{Xe}$ into the first $0^{+}$excited state, $0_{1}^{+}$, of ${ }^{136} \mathrm{Ba}$ was also investigated. The Q-value of this process is $878.8 \mathrm{keV}$, which is followed by the emission of two de-excitation $\gamma_{\mathrm{S}}$ with energies of $760.5 \mathrm{keV}$ and $818.5 \mathrm{keV}$. A boosted decision tree machine learning algorithm is employed to create a discriminator variable, which provides more power to constrain the backgrounds than the standoff distance alone. The event quantities used in this discriminator are the energy, multiplicity, sum energy of the de-excitation $\gamma_{\mathrm{s}}$, standoff distance, and the individual de-excitation $\gamma$ energies. No statistically significant evidence for this $\beta \beta$ decay is found, with a half-life sensitivity reach of $1.7 \cdot 10^{24} \mathrm{yr}$ at $90 \% \mathrm{CL}$. The profile likelihood scan results in a lower limit of $\mathrm{T}_{1 / 2}^{2 v\left(0^{+} \rightarrow 0_{1}^{+}\right)}>6.9 \cdot 10^{23} \mathrm{yr}[10]$.

The EXO-200 Phase-I data was used for other studies in addition to the search for $\beta \beta$ decays. The ion fraction and mobility of $\alpha$ and $\beta$ decay products in LXe were measured [11]. It is found that 
$76.4 \pm 5.7 \%$ of the ${ }^{214} \mathrm{~Pb} \beta$-decays produce ${ }^{214} \mathrm{Bi}^{+}$and $50.3 \pm 3.0 \%$ of the ${ }^{222} \mathrm{Rn} \alpha$-decays produce ${ }^{218} \mathrm{Po}^{+}$. The average velocity of these ions is observed to depend on the drift time. These results are particularly relevant for the development of a technique to $\operatorname{tag}{ }^{136} \mathrm{Ba}$, the daughter of the $\beta \beta$ decay in ${ }^{136} \mathrm{Xe}$, for the $\mathrm{nEXO}$ experiment [12]. Finally, the collaboration presented measurements of the transverse diffusion constant and drift velocity of electrons at drift fields between $20 \mathrm{~V} / \mathrm{cm}$ and $615 \mathrm{~V} / \mathrm{cm}[13]$.

\subsection{Phase-II Operation}

After the halt of underground access imposed by WIPP, EXO-200 was smoothly and successfully restarted in 2015. The detector levels of purity and internal radon contamination were excellent shortly after filling the TPC vessel with LXe in January 2016. Two major detector upgrades were pursued between February and April, 2016: the installation of improved front-end electronics, and installation of a Rn-suppressed air system.

The front-end readout system was upgraded to reduce both the coherent noise in the APD channels and the energy threshold for the V-wire channels. This effort followed the evidence from data that the detector energy resolution, as well as the analysis energy threshold, were limited by the coherent noise in the light readout channels. After an extensive investigation of the noise data and bench tests, it was decided to replace the APD boards with new preamp design less sensitive to the regulator noise. Moreover, ground adapters were installed to minimize ground currents between APD channels. Initial measurements indicate a reduction by a factor of 2.5 in the coheret noise between Phase-I and Phase-II data. Finally, the V-wire shaping times were optimized to lower the $2 \mathrm{D}$ reconstruction threshold.

The energy resolution substantially improved after the new front-end electronics. In Phase-I data, SS events in calibration runs with a ${ }^{228}$ Th source positioned near the cathode present a resolution $(\sigma / E)$ that varies between $\sim 1.5 \%$ and $\sim 2 \%$ at the ${ }^{208} \mathrm{Tl} \gamma$-line, $2615 \mathrm{keV}$, as shown in figure 1. This variation is assigned to that experienced by the coherent noise. It can be largely reduced using an offline denoising algorithm developed by the collaboration [14]. The corresponding denoised energy resolution is restricted to values below 1.6\%. With the electronics upgrade in Phase-II, the energy resolution immediately reduces to $1.38 \%$ without application of the offline denoising process. The performance of the detector is further optimized by increasing the cathode voltage from $8 \mathrm{kV}$ to $12 \mathrm{kV}$, i.e. a drift field change from $\sim 380 \mathrm{~V} / \mathrm{cm}$ to $\sim 576 \mathrm{~V} / \mathrm{cm}$. The detector has been operating stably under these conditions since June, 2016, with energy resolution reaching $1.28 \%$ at the Q-value.

Radon levels in the EXO-200 clean room was monitored during Phase-I running with a Rad7 detector and is averaged to be $\sim 7 \mathrm{~Bq} / \mathrm{m}^{3}$. In parallel, analysis of the physics data hints to some backgrounds of the $0 v \beta \beta$ search arising from radon in the air gap between the lead shielding and the outer cryostat vessel. Its exact amount is difficult to infer because of the degeneracies in the background models. Therefore, it was decided to purge radon from air in this location with an apparatus, referred to as the de-radonator, built and tested at the University of Massachussets, Amherst. Preliminary measurements show that the radon level in the air gap has been reduced by a factor of $\sim 10$, which is expected to remove this background contribution from future $0 v \beta \beta$ searches. 


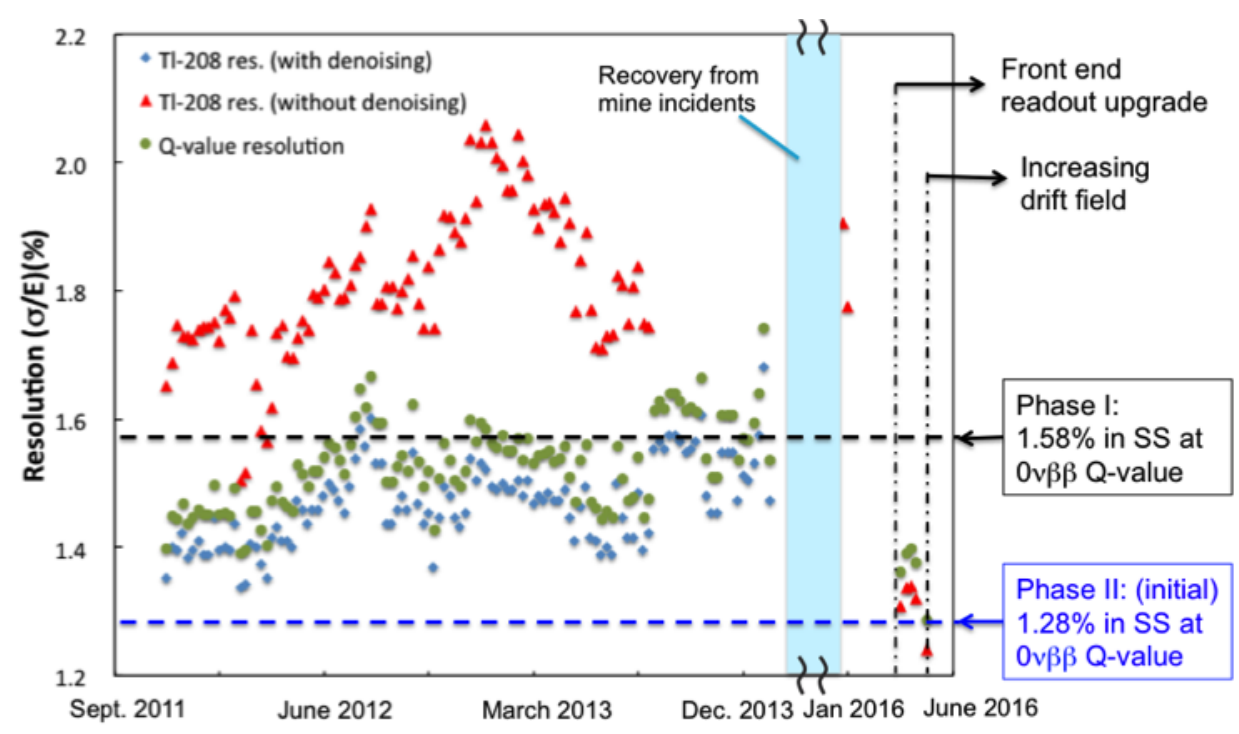

Figure 1: EXO-200 resolution for SS events versus time. This resolution is measured as a function of energy by fitting Monte Carlo simulation to calibration data [9].

In April, 2016, EXO-200 started physics DAQ in Phase-II. Studies accounting for these two hardwarde upgrades, as well as realistic analysis improvements that includes vetoing events arising from ${ }^{137} \mathrm{Xe}$ decays, project a sensitivity to the $0 v \beta \beta$ half-life of $5.7 \cdot 10^{25} \mathrm{yr}$ at $90 \% \mathrm{CL}$ using data from Phase-I and II. This value is similar to that obtained by the KamLAND-Zen experiment [15], thus expected to provide an important independent check of this result.

\section{The nEXO Experiment}

The next generation of $0 v \beta \beta$ experiments is expected to extend the experimental sensitivity of this search by about two orders of magnitude. This can be converted into a sensitivity to the effective Majorana mass that strongly depends on the nuclear model employed. The Majorana mass is limited at $\sim 15 \mathrm{meV}$ for the inverted hierarchy of neutrino masses as depicted in figure 2 .

Rooted in the success that EXO-200 has demonstrated, the nEXO collaboration is planning a detector with $\sim 5 \mathrm{t}$ of LXe. An intensive R\&D program in various fronts is underway to validate the concept of the nEXO detector. In particular, its resolution is expected to be $\sigma / E=1 \%$ at the Qvalue, which will be achieved primarily by a large photo-coverage using silicon photo-multipliers placed along the TPC barrel.

Sensitivity calculations for the nEXO experiment rely on detailed simulations of the detector in combination with background projections that are solidly grounded in existing EXO-200 and radioassay data. The analysis employed in these calculations is that established in the EXO-200 searches. After 10 years of data, the projected nEXO half-life sensitivity is $9.5 \cdot 10^{27} \mathrm{yr}$ at $90 \% \mathrm{CL}$ as shown in figure 3 [16].

\section{Acknowledgments}

EXO-200 and nEXO are supported by DoE and NSF (USA), NSERC (Canada), SNF 


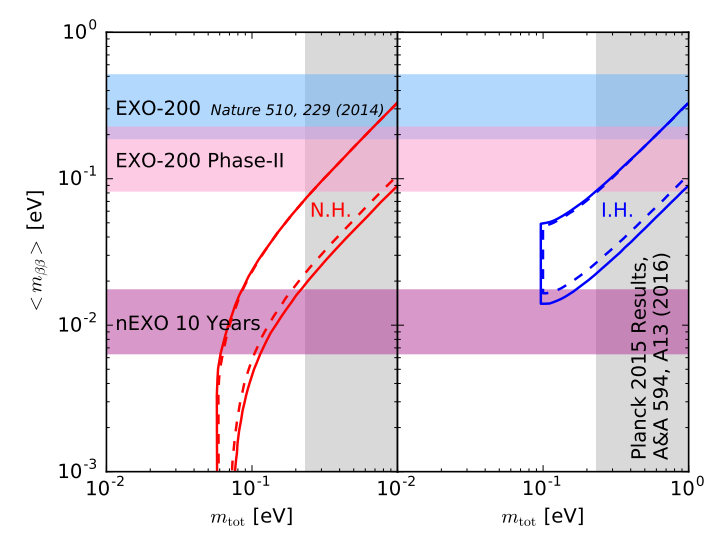

Figure 2: Sensitivity projections to the Majorana neutrino mass $\left(m_{\beta \beta}\right)$ for the EXO-200 and nEXO experiments. The bands are regions as a function of the sum of neutrino masses allowed for the normal (left) and inverted (right) hierarchies.

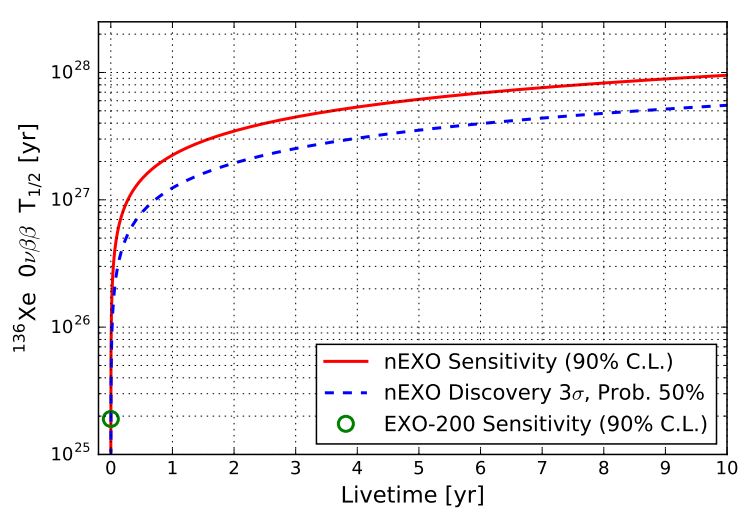

Figure 3: Projected nEXO sensitivity and discovery potential to the $0 v \beta \beta$ half-life at $90 \% \mathrm{CL}$ as a function of the detector livetime.

(Switzerland), NRF (South Korea), RFBR (Russia), and the DFG Cluster of Excellence Universe (Germany).

\section{References}

[1] J. Schechter and J. W. F. Valle Phys. Rev. D 252951 (1982)

[2] M. Redshaw, E. Wingfield, J. McDaniel, and E. G. Myers Phys. Rev. Lett. 98053003 (2007)

[3] N. Ackerman et al. [EXO-200 Collaboration] Phys. Rev. Lett 107212501 (2011)

[4] J. B. Albert et al. [EXO-200 Collaboration] Phys. Rev. C 89015502 (2014)

[5] J. B. Albert et al. [EXO-200 Collaboration] Nature 510229 (2014)

[6] M. Auger et al. [EXO-200 Collaboration] J. Instrum. 7 P05010 (2012)

[7] J. B. Albert et al. [EXO-200 Collaboration] Phys. Rev. C 92015503 (2015)

[8] J. B. Albert et al. [EXO-200 Collaboration] JCAP 04029 (2016)

[9] J. B. Albert et al. [EXO-200 Collaboration] Phys. Rev. D 90092004 (2014)

[10] J. B. Albert et al. [EXO-200 Collaboration] Phys. Rev. C 93035501 (2016)

[11] J. B. Albert et al. [EXO-200 Collaboration] Phys. Rev. C 92045504 (2015)

[12] B. Mong et. al. [nEXO Collaboration] Phys. Rev. A 91022505 (2015)

[13] J. B. Albert et al. [EXO-200 Collaboration] Measurement of the Drift Velocity and Transverse Diffusion of Electrons in Liquid Xenon with the EXO-200 Detector [physics/1609.04467]

[14] C. G. Davis et al. [EXO-200 Collaboration] J. Instrum. 11 P07015 (2016)

[15] A. Gando et al. [KamLAND-Zen Collaboration] Phys. Rev. Lett 117082503 (2016)

[16] C. Licciardi, The Sensitivity of the nEXO Experiment to Majorana Neutrinos, in proceedings of XXVII International Conference on Neutrino Physics and Astrophysics 